\title{
Redécouverte de Prionotropis appula en Grèce occidentale (Orth.
}

\section{Pamphagidae)}

Antoine Foucart, Philippe Ponel

\section{Citer ce document / Cite this document :}

Foucart Antoine, Ponel Philippe. Redécouverte de Prionotropis appula en Grèce occidentale (Orth. Pamphagidae). In: Bulletin de la Société entomologique de France, volume 104 (5), décembre 1999. pp. 465-466;

https://www.persee.fr/doc/bsef_0037-928x_1999_num_104_5_16617

\section{Ressources associées :}

Prionotropis appula

Fichier pdf généré le 30/09/2019 
1990. - Le genre Manduca Hübner (1807) en Guyane française. Approche écologique et remarques systématiques (Lepidoptera Sphingidae). Bulletin de la Société Sciences Nat, 66 : 1-8.

— 1992. - Les Aleuron Boisduval (1870) en Guyane française (Lepidoptera Sphingidae). Bulletin de la Société Sciences Nat, 73 : 13-16.

1993. - Systématique et répartition des espèces du groupe d'Hyles lineata (Fabricius). Lambillionea, 93 : 156-166.

_ 1994. - Description d'un nouveau Sphingidae de Guyane française: Xylophanes colinae n. sp. (Lepidoptera Sphingidae). Lambillionea, 94: 137-141.

1996. - Les genres Pachygonia Fletcher, Nyceryx Boisduval et Perigonia Herrich-Schäffer en Guyane française (Lepidoptera, Sphingidae). Lambillionea, 96:342-350.

HAXAIRE J. \& RASPLUS J.-Y., 1986. - Contribution à la connaissance des Sphingidae de Guyane française. $1^{\mathrm{c}}$ partie. Bulletin de la Société entomologique de France, 91 (9-10): 275-285.

- 1987. - Contribution à la connaissance des Sphingidae de Guyane française. $2^{\mathrm{e}}$ partie. Bulletin de la Société entomologique de France, 92 (1-2): 45-55.

KISLEV M.E., KRAVIZ Z. \& LORCH J., 1972. - A study of hawkmoth pollination by palynological analysis of the proboscis. Israël Journal of botany, $21: 57-75$.

LiCHY R., 1976. - La mission d'exploration entomologique du Muséum National d'Histoire Naturelle en Guyane française: Sphingidae (Lep.). Bulletin de la Société entomologique de France, 80 (910): 271-275.

LinharT Y. B. \& MENDENhall J. A., 1977. - Pollen dispersal by hawkmoths in Lindenis rivalis Benth. population in Belize. Biotropica, 9: 763-774.

MILLER R. B., 1981. - Hawkmoths and geographic patterns of floral variation in Aquilegia caerulea. Evolution, 35: 763-774.

\section{Antoine FOUCART et Philippe PONEL. - Redécouverte de Prionotropis appula en Grèce occidentale (Orth. Pamphagidae)}

Cette note confirme la présence en Grèce occidentale de Prionotropis appula (Costa, 1836). Cette espèce d'Acridien est un Pamphagidae Akicerinae décrit des Pouilles qui se rencontre dans toute l'Italie méridionale, fig. 1 (HARZ, 1975); elle est connue d'Épire par des exemplaires récoltés au siècle dernier (BRUNNER, 1882) mais n'avait pas été observée depuis plus d'un siècle (WILLEMSE, 1984).

L'un d'entre nous (Ph. P.) l'a redécouvert fortuitement dans la région d'Ioanina (Épire), confirmant ainsi la citation de Brunner von Wattenwyl. Deux $\delta$ et une $q$ ont été récoltés le 10.VI.1997 au bord de la route qui mène à Aristi, dans une garrigue à Phlomis fructicosus, parsemée de bosquets de chênes et de prairies sèches correspondant certainement à d'anciennes cultures. Les exemplaires récoltés sont des imagos immatures, à téguments mous, et sont conservés au Muséum national d'Histoire naturelle, à Paris, et dans la collection Ph. Ponel.

L'habitus et les génitalia des exemplaires récoltés en Grèce ne diffèrent en rien de ceux d'Italie. UVAROV (1923), lors de sa révision du genre Prionotropis, faisait remarquer qu' «Il est possible que l'Épire soit habitée par une race locale distincte de celle du Sud de l'Italie, mais je n'ai vu qu'un exemplaire de cette provenance, très semblable à ceux d'Italie.» Le faible nombre d'exemplaires récoltés ne permet pas d'infirmer ou de confirmer son hypothèse. En l'état actuel de nos connaissances, nous ne pouvons savoir si les populations grecques et italiennes se différencient au niveau subspécifique. Des analyses plus poussées au plan génétique apporteraient sans doute un éclairage nouveau sur ce problème.

Cette note est l'occasion de rappeler que l'origine du peuplement des Orthoptères de l'Italie est assez complexe. Dans le cas de $P$. appula, tout laisse à penser que cette espèce s'est installée dans le sud de l'Italie au cours de la régression marine et de l'extension des terres 


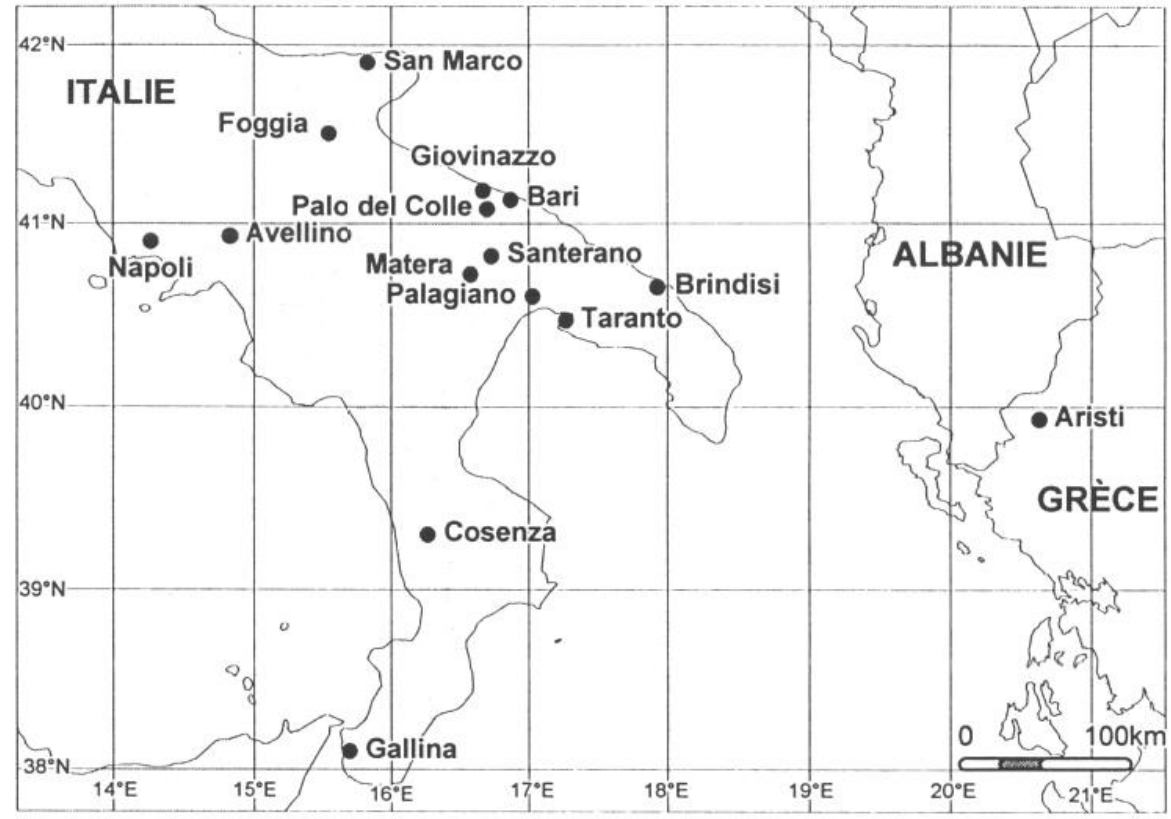

Fig. 1. - Répartition de Prionotropis appula d'après HARZ (1975).

émergées qui a eu lieu entre le Miocène et le Pliocène (le Pontien) (LA GrECA, 1959, 1985). $P$. appula aurait alors atteint en Italie la limite occidentale de sa répartition lors de l'assèchement de la mer Égée. Ce type de répartition transégéenne est bien connu pour deux reptiles: un gecko (Cyrtodactylus kotschyi) et une couleuvre (Elaphe situla), ainsi que pour un arbre, le chêne des Balkans (Quercus macrolepis) (BRUNO, 1986), mais est encore mal connu pour les insectes.

Nous incitons nos collègues orthoptéristes à rechercher plus particulièrement cette espèce lors de leurs voyages. Prionotropis appula a certainement une répartition plus vaste (orientale?) qu'on ne le suppose. Il serait intéressant de mieux connaître la répartition actuelle de cette espèce et de voir s'il existe une zone de contact avec $P$. hystrix (Germar, i 817), autre espèce présente dans les Balkans.

\section{AUTEURS CITÉS}

BRUNNER VON WATTENWYL C., 1882. - Prodromus der Europäischen Orthopteren. Englemean, Leipzig: $466 \mathrm{p}$.

BRUNO S., 1986. - Guida a Tartarughe e sauri d'Italia. Giunti Martello, Firenze. 255 p.

IIARZ K., 1975. - The Orthoptera of Europa, tome 2, vol. 11. Dr. W. Junk, La Hague : 939 p.

LA GRECA M., 1959. - L'Ototterofauna puglieuse ed il suo signicato biogeografico. Memorie di Biogeografia Adriatica, IV: 33- 170.

— 1985. - Origine des Orthoptères de la faune d'Italie. Mitteilungen der Deutschen Gesellschaft für Allgemeine und Angewandte Entomologie, $4: 223-225$.

Uvarov B., 1923. - Sur les races géographiques du Prionotropis hystrix Germ. (Orth. Acrididae) Annales de la Société entomologique de France (1922), XCI : 245-248.

WILLEMSE F., 1984. - Fauna Graeciae, catalogue of the orthoptera of Greece. Hellenic Zoological Society, Athens : 275 p.

(A. F. : CIRAD, Protection des Cultures, unité d'Acridologie, BP 5035, F-34032 Montpellier cedex 1, <antoine.foucart@cirad.fr> ;

Ph. P. : Faculté des Sciences et Techniques de Saint-Jérôme, laboratoire de Botanique historique et Palynologie (Boite 451), F-13397 Marseille Cedex 20) 